Fachbereich 5

Wirtschaftswissenschaften, Wirtschaftsinformatik und Wirtschaftsrecht

Volkswirtschaftliche Diskussionsbeiträge

Discussion Papers in Economics

No. 131-09

July 2009

Rüdiger Pethig and Christian Wittlich

Interaction of carbon reduction and green energy promotion in a small fossil-fuel importing economy 


\section{Universität Siegen}

Fachbereich 5

Wirtschaftswissenschaften, Wirtschaftsinformatik und Wirtschaftsrecht

Fachgebiet Volkswirtschaftslehre

Hölderlinstraße 3

D-57068 Siegen

Germany

http://www.uni-siegen.de/fb5/vwl/

ISSN 1869-0211

Available for free from the University of Siegen website at http://www.uni-siegen.de/fb5/vwl/research/diskussionsbeitraege/

Discussion Papers in Economics of the University of Siegen are indexed in RePEc and can be downloaded free of charge from the following website:

http://ideas.repec.org/s/sie/siegen.html 


\title{
Interaction of carbon reduction and green energy promotion in a small fossil-fuel importing economy
}

\begin{abstract}
We study the incidence of carbon-reduction and green-energy promotion policies in a general equilibrium small open economy that depends on imports of fossil fuels. The focus is on mixed policies that are either price based (emissions taxes and producer price subsidies for green energy) or quantity based (schemes of trading emissions and green certificates). Policy instruments directed head-on toward promoting green energy are shown to also reduce carbon emissions and vice versa but the direct effects are stronger than the side effects, the more so, the greater is the elasticity of substitution in consumption between energy and consumption goods. We calculate the effects of variations in individual policy parameters on all endogenous variables, among them the energy price and the welfare costs. We also determine the impact of exogenous fossil-fuel price shocks on the economy.
\end{abstract}

JEL Classifications: Q42, Q43, Q48, Q54

Keywords: carbon reduction, green energy promotion, policy mix, interaction of policies 


\section{The problem}

Many countries in the world, notably the Annex I countries of the Kyoto Protocol, take action to curb carbon dioxide emissions, and many OECD countries have policies to promote energy from renewable energy sources (OECD/IEA 2008). Our focus is on countries pursuing a mix of both policies and depending on imports of fossil fuels like e.g. most European countries. Conceptually, carbon emissions are reduced by making them more expensive and green energy is stimulated by raising its producer price. However, increasing the price of carbon emissions not only reduces emissions (direct effect), but also gives green energy a competitive edge over black energy and hence indirectly promotes green energy production. Likewise, subsidizing green energy not only boosts the production of green energy (direct effect), but also makes green energy more competitive vis-à-vis black energy, which indirectly curbs carbon emissions. The present paper aims to explore rigorously the interaction of these mixed strategies in a simple model of a small open economy that imports all of its fossil fuel input and produces black as well as green energy.

While carbon-cutting policies are widely seen as an effective means to cope with the global negative climate-change externality, the rationale for supporting green energy is less consensual. Along with several national governments ${ }^{1}$, the European Commission (Com (2007) 1) acknowledges serious energy challenges concerning security of supply and import dependence and argues that the promotion of renewable energies plays a part in securing energy supply. The Renewable Energies Roadmap is to enable the EU to meet the 'twin objectives' of increasing security of energy supply and reducing greenhouse gas emissions.

In the present paper, we do not include in our formal modeling the (potential) external social benefits of the policies under scrutiny, since our focus is policy incidence taking real world policy targets as given. Rather than providing a full cost-benefit account of these policies, we focus on assessing their (welfare) costs relative to the no-regulation base line. Specifically, we will consider

(i) quantity-based mixed policies with explicitly established quantitative emission targets (standards or caps) and green energy targets (standards) that are implemented by means of appropriate 'prices',

(ii) and price-based mixed policies combining an emissions tax with a subsidy on the producer price of green energy in the absence of quantity standards.

Usually, taxes and subsidies are considered to be political prices that are means rather than ends. However, in practice they may come close to be targets in their own right when no quantity standards exist and short-term changes of rates are unlikely or even infeasible. We will take that view when we investigate price-based mixed policies. Although quantity-based policies play an increasing role in many countries, and hence deserve special attention, analyzing mixed price-based policies is still relevant not least because their study will turn out below to provide a sound analytical foundation for the exploration of quantity-based mixed policies $^{3}$. When standards are defined, they are implemented by means of political or market

\footnotetext{
${ }^{1}$ One of several purposes of the German Renewable Energy Act (Bundesregierung 2008, §1) is to reduce the dependence on energy imports.

${ }^{2}$ Baumol and Oates (1971) are among the first to use in their analysis standards as quantitative upper bounds for emissions without explicit reference to those standards' net welfare implications. They defend their approach on practical grounds arguing that such standards are "somewhat arbitrary" representing the decision maker's subjective evaluation.

${ }^{3}$ Emissions caps are in operation in the Annex I countries of the Kyoto Protocol with the Kyoto cap of the EU being split up in member state caps. Binding green energy standards are envisioned for all EU member states since the EU has set itself the target of increasing the share of renewables in energy use to 20\% by 2020 (EU
} 
prices, i.e. either by means of emissions taxes and green energy subsidies or, alternatively, by means of emissions trading schemes and green certificate schemes ${ }^{4}$. In our simple stylized model, both these types of price-and-standard policies will turn out to be equivalent (see footnote 15 below). Due to their growing importance in recent years, we will mainly focus on those quantity-based mixed policies that combine an emissions trading scheme with a green certificates scheme.

We will analyze the mixed policies outlined above to answer the following questions:

(a) How do exogenous changes in individual policy parameters (prices or quantities) affect the endogenous policy variables, the equilibrium allocation and prices, and the representative consumer's utility (= welfare)?

(b) Given the - expected - result that strategies of pricing emissions [of subsidizing the producer price of green energy] are relatively more effective in reducing emissions [in promoting green energy] than in promoting green energy [in reducing emissions]: What are the determinants of relative effectiveness?

(c) What is the impact of (exogenous) price shocks in the world market for fossil fuel on the economy, when the regulator keeps her mixed policy unchanged after the shock?

We will show how the allocative displacement effects of exogenous changes in individual policy parameters depend on whether the policy is price-based or quantity-based. In all comparative static exercises, we calculate the sign of the change in utility (welfare), which turns out to be unambiguous in most cases. That allows us to determine, in qualitative terms, the welfare loss associated to the mixed policies and provides policy makers with the information what the minimum level of potential external social benefits of the policy (not included in our formal model) must be in order to reap a net social benefit from that policy.

Having characterized the differences in policy effectiveness (point (b) above), we show how the interaction of supply and demand conditions determines the extent to which the policy effectiveness differs. For economies heavily relying on imports of fossil fuels the answer to the question (c) will be particularly important, since during the past years the world economy experienced massive increases in the price of fossil fuels and further price hikes are likely in the medium and long term. Obviously, the effects of a price shock will depend on the kind of mixed policy chosen. We show, in particular, how the fossil fuel price shock changes the energy price in the economy under consideration.

The interaction of policies aiming to reduce carbon emissions and to promote green energy has already received much attention in the literature. To our knowledge, the first analytical approach is due to Jensen and Skytte (2003). Further pertaining studies are, among others, Sorrell and Sijm (2003), EC Directorate-General Environment (2005), Federal Environmental Agency (2007), Abrell and Weigt (2008), Bye and Bruvoll (2008) and Linares et al. (2008). Bode and Groscurth (2006), Fischer (2006) and Rathmann (2007) focus especially on the impact of mixed policies on electricity prices. Gonzales (2007) provides an informative survey on analytical and numerical studies. Some of the extant analytical approaches are richer than our model in institutional detail but they provide limited insights, in our view, as they are partial equilibrium, rely exclusively on graphical analysis, employ parametric functional forms or

climate and energy package adopted by the European Parliament in December 2008). Mixed policies consisting of a quantity-based approach (emissions cap and trading) and a price-based approach are also found in practice (e.g. in Germany where we have an emissions cap and a feed-in tariff scheme). Yet analyzing such concepts does not yield major additional insights.

${ }^{4}$ We do not distinguish between green and white certificates because there would be no noteworthy difference in our simple model. See for example Bye and Bruvoll (2008) for a distinction between those systems. 
lack theoretical rigor. Closest to our approach is the model of Jensen and Skytte (2003, 2004). While they employ a partial equilibrium model, we choose a general equilibrium approach of a small open economy using general functional forms and a more structured setup. There are several further distinguishing features between our model and that of Jensen and Skytte. General rather than parametric functional forms; a representative consumer who consumes energy as well as a consumption good; a domestic input 'capital' used in the production of both green energy and the consumption good; imported fossil fuel, which the country needs to pay for with some amount of the consumption good it has produced.

The paper is organized as follows. Section 2 introduces the formal model. In Section 3, we lay the analytical foundation for most of the paper and investigate the incidence of price-based mixed policies. The comparative static analysis of the general equilibrium model provides insights into the effects of changes in policy parameters and the price of fossil fuel on the allocation, on prices and on welfare. Section 4 proceeds with extending the comparative statics to quantity-based mixed policies and the results are compared with the incidence of pricebased mixed policies from Section 3. Section 5 concludes.

\section{The economy}

Consider the economy of a small open country that produces the amount $x_{s}$ of a consumer good and the amount $z$ of energy. The production function of the consumer $\operatorname{good}(\operatorname{good} X)$ is

$$
x_{s}=Q^{x}\left(k_{x}\right)
$$

where $k_{x}$ represents capital input. The energy produced,

$$
z=b+g \text {, }
$$

consists of black energy, $b$, generated from fossil fuel, $e$, via the production function

$$
b=Q^{b}(e)
$$

and of energy, $g$, called green energy, generated from capital input, $k_{g}$, via the production function

$$
g=Q^{g}\left(k_{g}\right) .
$$

All production functions are assumed to be strictly concave. The economy's capital endowment is

$$
k=k_{g}+k_{x} \text {. }
$$

Energy and capital are traded on domestic markets at prices $p_{z}$ and $p_{k}$, respectively, while the consumer good and fossil fuel are traded on world markets at fixed prices $p_{x} \equiv 1$ and $p_{e}$. All fossil fuel needs to be imported, and the country pays for those imports by exporting the consumer good. Accordingly, the trade balance is

$$
x_{s}-x-p_{e} e=0,
$$

where $x_{s}$ is the supply of and $x$ is the demand for the consumer good. 
The representative consumer derives utility $U(x, z)$ from the consumer good and energy. She maximizes her utility by a suitable choice of consumption $(x, z)$ keeping her total expenditure $x+p_{z} z$ in line with her income. The resultant standard condition for optimal consumption is

$$
\frac{U_{z}}{U_{x}}=p_{z}
$$

Note that by assumption neither the producers nor the consumer is affected by negative externalities that would provide a rationale for controlling emissions and for promoting green energy. As pointed out in the introduction, we exclude the benefits of regulation and thus do not deal with the issue of optimal or efficient regulation.

For simplicity, we represent each production sector by a single aggregate firm. Each of these firms is assumed to maximize its respective profits $\pi_{b}:=p_{z} Q^{b}(e)-\left(p_{e}+t\right) e, \pi_{x}:=Q^{x}\left(k_{x}\right)$ $p_{k} k_{x}$, and $\pi_{g}:=\left(p_{z}+s\right) Q^{g}\left(k_{g}\right)-p_{k} k_{g} . s \geq 0$ denotes the rate of a subsidy on green energy and $t \geq 0$ is the rate of a tax on fossil fuel. The tax on fossil fuel is equivalent to a carbon emissions tax because carbon emissions are (almost) proportional to fossil fuel burned in the process of producing black energy.

If the regulator does not pursue quantitative targets, as we will assume for the time being and throughout the next Section 3, we deal with a tax-subsidy policy. Quantity-based mixed policies will be analyzed later in Section 4.

The standard first-order conditions for maximizing profits are

$$
\begin{aligned}
& p_{z} Q_{e}^{b}(e)=p_{e}+t, \\
& \left(p_{z}+s\right) Q_{k}^{g}\left(k_{g}\right)=p_{k}, \\
& Q_{k}^{x}\left(k_{x}\right)=p_{k} .
\end{aligned}
$$

We complete the description of the model by specifying the consumer's budget constraint as

$$
\pi_{b}^{*}+\pi_{g}^{*}+\pi_{x}^{*}+p_{k} k+t e-s g=x+p_{z} z
$$

where $\pi_{b}^{*}, \pi_{g}^{*}, \pi_{x}^{*}$ are the firms' maximum profits at given prices and policy parameters. ${ }^{5}$

For any given tax-subsidy policy $(\bar{s}, \bar{t})$, a competitive equilibrium of the economy (1) - (11) is a state where firms maximize profits, the consumer maximizes her utility subject to (11) and where prices $p_{z}$ and $p_{k}$ are market clearing. Note that equation (11) is redundant because inserting into (11) the profits as defined above yields (6). Therefore the equations (1) (10) uniquely determine the equilibrium prices, $\left(p_{k}, p_{z}\right)$, and the equilibrium allocation of resources, $\left(e, b, g, k_{g}, k_{x}, x, x_{s}, z\right)$. It is obvious from the definition of equilibrium that the regulator's choice of policy $(\bar{s}, \bar{t})$ fully determines the equilibrium prices and the equilibrium

\footnotetext{
${ }^{5}$ The rate of subsidy $s$ can easily be replaced by a feed-in tariff or premium. For that purpose, one would only have to drop the term (-sg) in (11) and change the definition of profit in the black energy sector from $\pi_{b}$ into $\tilde{\pi}_{b}:=\pi_{b}-s g$.
} 
allocation. We now proceed to exploring the impact of variations in the tax and the subsidy on equilibrium prices and quantities.

\section{Incidence of price-based mixed policies: carbon emissions taxes com- bined with green subsidies}

\subsection{Levels of emissions and green energy attainable through tax-subsidy policies}

In this subsection, the focus is on a tax-subsidy policy with rates $(\bar{s}, \bar{t})$. We begin with exploring how the equilibrium values of green and black energy change when the rates $(\bar{s}, \bar{t})$ are varied. We expect - and will confirm - that the subsidy stimulates green energy production and that the tax curbs carbon emissions and black energy production. Yet it is less clear what the side effects are of the a change in the emissions tax on green-energy production and what the side effects are of a change in the green subsidy on carbon emissions. The comparative-static analysis yields ${ }^{6}$

Result 1. Denote by $e=E(s, t)$ and $g=G(s, t)$ the equilibrium emissions and the equilibrium green energy, respectively, when the regulator fixes the tax and subsidy at rates $(s, t)$.

(i) $\quad E_{s}<0$ and $E_{t}<0$, i.e. the carbon tax curbs carbon emissions, and it promotes greenenergy production as well.

(ii) $G_{s}>0$ and $G_{t}>0$, i.e. the green energy subsidy stimulates green energy production, and it curbs carbon emissions as well.

According to Result 1, the tax as well as the subsidy is capable of promoting both goals. The driving force for that result is the differential response of the equilibrium energy price, $p_{z}=P^{z}(s, t)$, to changes in the tax and subsidy. Its partial derivatives exhibit the signs $P_{t}^{z}>0$ and $P_{s}^{z}<0$. Therefore, an increase in the tax rate raises the energy price and with it the green energy producers' revenues which, in turn, stimulates green energy production. ${ }^{7}$ Conversely, increasing the subsidy rate reduces the energy price and thus discourages the production of black energy. ${ }^{8}$ Hence, the induced change in the energy price $p_{z}$ turns out to be the transmission channel for the side effects of variations in the tax and subsidy.

Although Result 1 provides useful basic information, it does not answer the question as to how strong the side effect $G_{t}>0\left[E_{s}<0\right]$ is relative to the direct effect $G_{s}>0\left[E_{t}<0\right]$. To gain further insight we will characterize the set of all pairs of energy $[E(s, t), G(s, t)]$ generated by varying the policy $(s, t)$ over $\mathbb{R}_{+}^{2}$. Suppose first, the regulator either fixes $s=\bar{s} \geq 0$

\footnotetext{
${ }^{6}$ All results are proved in the Appendix.

${ }^{7}$ The production of black energy is stimulated by this effect as well. However, that expansive effect on black energy is overcompensated by the contractive effect of increased tax payment (factor costs).

${ }^{8}$ One can show that using an argument similar to that which we have put forward in the preceding footnote.
} 
and varies $t$ or she fixes $t=\bar{t} \geq 0$ and varies $s$. We describe these policies in a formal way by introducing the functions $\Gamma^{\bar{t}}$ and $\Gamma^{\bar{s}}$ defined by

$$
g=\Gamma^{\bar{s}}(e, \bar{s}):=G\left[\bar{s}, \tilde{E}^{t}(e, \bar{s})\right] \text { and } g=\Gamma^{\bar{t}}(e, \bar{t}):=G\left[\tilde{E}^{s}(e, \bar{t}), \bar{t}\right],
$$

where $\tilde{E}^{t}$ and $\tilde{E}^{s}$ are inverse functions defined by the equivalences

$$
" t=\tilde{E}^{t}(e, \bar{s}) \Leftrightarrow e=E(\bar{s}, t) " \text { and " } s=\tilde{E}^{s}(e, \bar{t}) \Leftrightarrow e=E(s, \bar{t}) " .
$$

For convenience, we refer to the function $\Gamma^{\bar{s}}$ as the "s= $\bar{s}$ function" and to the function $\Gamma^{\bar{t}}$ as the " $t=\bar{t}$ function". These functions are useful tools for studying the impact of tax-subsidy policies on black and green energy, which is characterized in

Result 2. Let $\bar{s} \geq 0$ and $\bar{t} \geq 0$ (not too large ${ }^{9}$ ) be given.

(i) The $t=\bar{t}$ function $\Gamma^{\bar{t}}$ and the $s=\bar{s}$ function $\Gamma^{\bar{s}}$ as defined in (12) have the point $[E(\bar{s}, \bar{t}), G(\bar{s}, \bar{t})]$ in common. Their derivatives with respect to $e, \Gamma_{e}^{\bar{t}}$ and $\Gamma_{e}^{\bar{s}}$, are negative.

(ii) Except for a polar case (see below) the derivative with respect to $e$ of the $t=\bar{t}$ function is greater in absolute value than that of the $s=\bar{s}$ function, i.e. the difference $\left|\Gamma_{e}^{\bar{t}}(e, \bar{t})\right|-\left|\Gamma_{e}^{\bar{s}}(e, \bar{s})\right|$ is positive for all $e<E(\bar{s}, \bar{t})$.

Figure 1 derives a negatively sloped $s=\bar{s}$ curve for some arbitrarily chosen (but not too large) $\bar{s} \geq 0$. It illustrates, in particular, that the points on the $s=\bar{s}$ curve $A B$ from $A$ toward $B$ are characterized by increasing tax rates. Since $\operatorname{sign} E_{s}(s, \bar{t})=\operatorname{sign} E_{t}(\bar{s}, t)$ and $\operatorname{sign} G_{s}(s, \bar{t})$ $=\operatorname{sign} G_{t}(\bar{s}, t)$, the $t=\bar{t}$ curves can be derived in a similar way and have a similar shape as the $s=\bar{s}$ curve $A B$ in Figure 1 .

Figure 2 is a modified and extended version of Figure 6 in Jensen and Skytte (2003). It illustrates that each $\mathrm{s}=\bar{s}$ curve and $\mathrm{t}=\bar{t}$ curve has one point in common (Result $2 \mathrm{i}$ ). For example, the $s=0$ curve and the $t=0$ curve in Figure 2 obviously meet in point $A .{ }^{10}$ Combine this observation with the finding of Result $2 \mathrm{ii}$ that the $s=0$ curve is steeper than the $t=0$ curve. The obvious implication is that $\Gamma^{\bar{s}}(e, s=0)<\Gamma^{\bar{t}}(e, t=0)$ for all $e<E(0,0)$.

We have thus established that the $t=0$ curve $A H$ begins in point $A$ as does the $s=0$ curve $A D$ and that the former lies above - and is steeper than - the latter. Although the points $D$ and $H$ have no practical relevance (because they imply $e=0$ ), their discussion is helpful for understanding Figure 2. In both points, $D$ and $H$, no black energy is produced $(e=0)$. However, in point $D$ green energy is produced without any subsidy, whereas in point $H$ the green subsidy is so high that the economy's total capital endowment is allocated to the production of green energy implying that the consumption good $X$ is not produced anymore. In that regard, our

\footnotetext{
${ }^{9}$ This qualification is meant to exclude corner solutions, i.e. those cases in which the tax rate or the subsidy rate is so large as to dry up fossil energy production altogether.

${ }^{10}$ Similarly, the point $C$ in Figure 2 is characterized by $\left(s=0, \mathrm{t}=t_{2}\right)$ because in point $C$ the $s=0$ curve and the $\mathrm{t}=t_{2}$ curve have a point in common.
} 
Figure 2 differs markedly from Figure 2 in Jensen and Skytte (2003) who have drawn the line $A D$ as a strictly convex line in such a way that the point $D$ coincides with the point $H$.

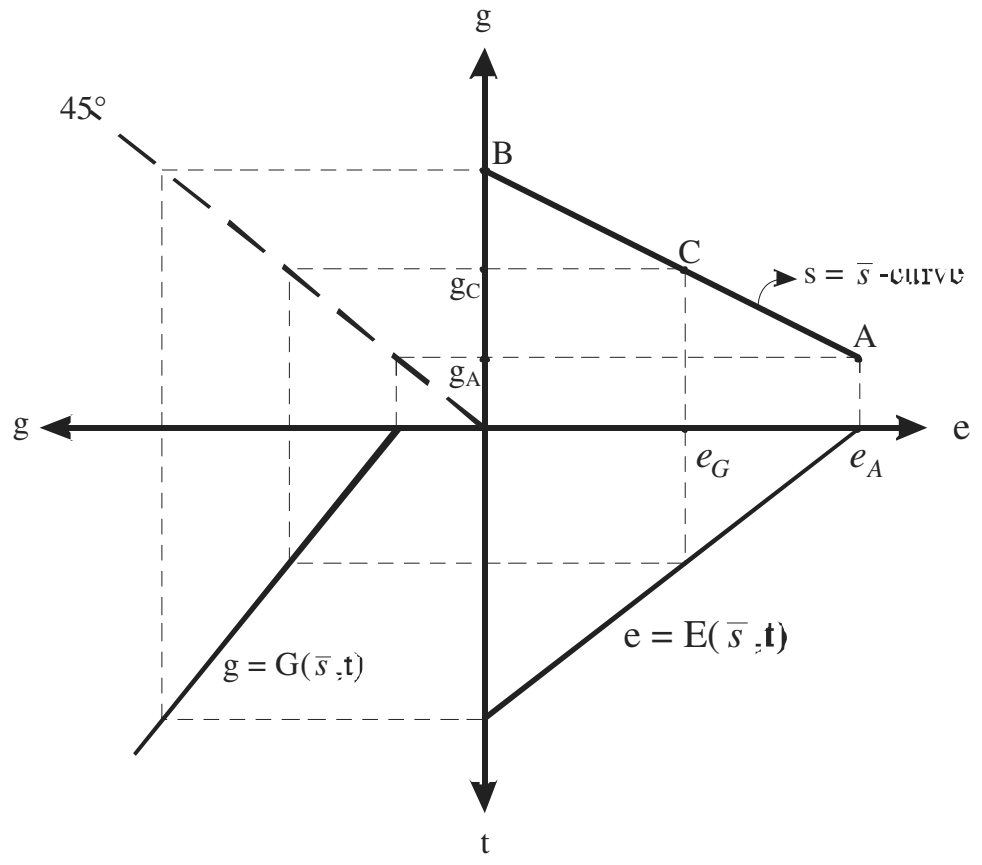

Figure 1: Varying the tax rate $t$ while the subsidy rate $s=\bar{s}$ is kept constant

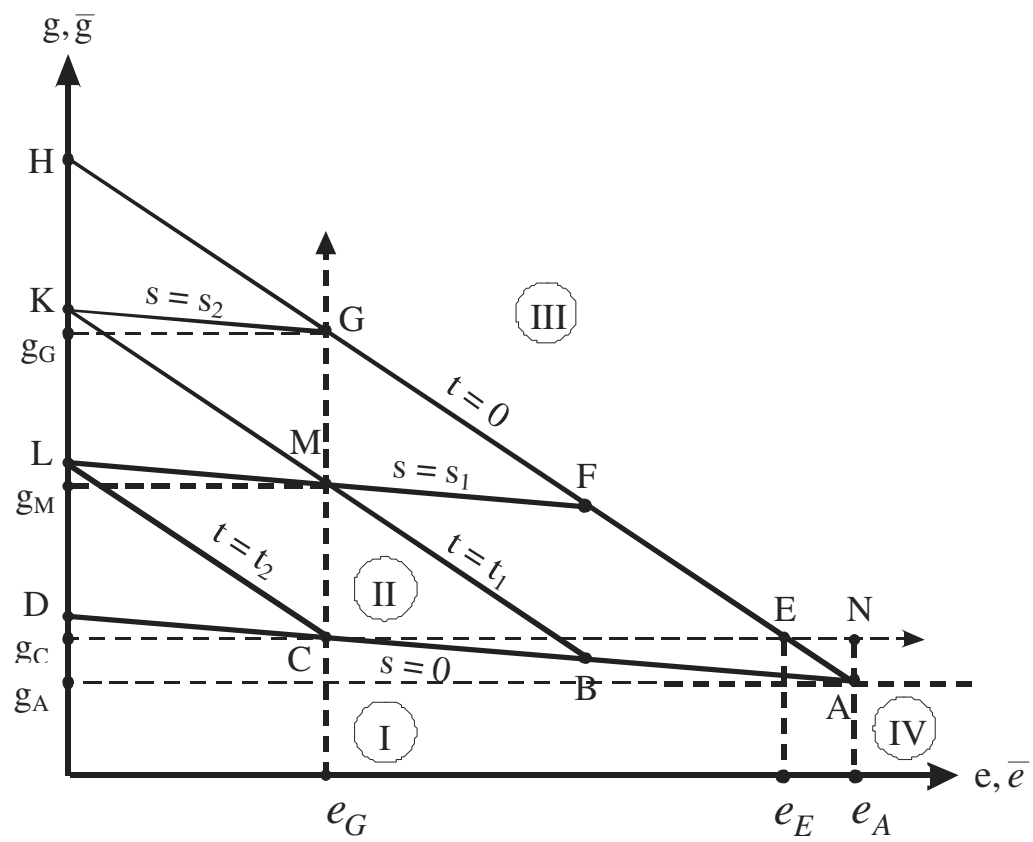

Figure 2: Allocations $(e, g)$ of emissions and green energy attained through alternative tax-subsidy policies $\left(0<s_{1}<s_{2}\right.$ and $\left.0<t_{1}<t_{2}\right)$ 
The lines $F L$ and $G K$ are also $s=\bar{s}$ curves with $\bar{s}=s_{1}$ along $F L$ and $\bar{s}=s_{2}$ along $K G$, satisfying $0<s_{1}<s_{2}$. Moreover, the lines $A H, B K$ and $C L$ are $t=\bar{t}$ curves with $\bar{t}=0$ along $A H$, $\bar{t}=t_{1}$ along $B K$ and $\bar{t}=t_{2}$ along $C L$, satisfying $0<t_{1}<t_{2}$. Note also that $t$ increases along $A D$ from $A$ toward $D$, along $F L$ from $F$ toward $L$ and along $G K$ from $G$ toward $K$. Likewise, $s$ increases along $A H$ from $A$ toward $H$, along $B K$ from $B$ toward $K$, and along $C L$ from $C$ toward $L$.

That the $t=\bar{t}$ curve lies above the $s=\bar{s}$ curve conforms to our intuition. To see the implication of that observation, suppose the regulator sets $s=0$ and chooses some tax rate $t_{C}>0$ such that $E\left(0, t_{C}\right)=e_{C}$ in Figure 2. The green energy produced is then $g_{C}$ which corresponds to the point $C$ on the $s=0$ curve. Consider now an alternative policy in which the regulator sets $t$ $=0$ and chooses the subsidy rate $s_{G}>0$ such that $G\left(s_{G}, 0\right)=g_{G} . s_{G}$ has been chosen deliberately in order to make the resultant emissions equal to $E\left(s_{G}, 0\right)=e_{C}$ as under the policy $\left(s=0, t_{C}\right)$. While the policies $\left(s=0, t_{C}\right)$ and $\left(s_{G}, t=0\right)$ both lead to the same emissions level $e_{C}$, the level of green energy generated under policy $\left(s_{G}, t=0\right)$ is higher than under policy $\left(s=0, t_{C}\right)$. This means that the subsidy is more effective than the tax in promoting green energy. Along similar lines, one can show that the tax is more effective than the subsidy in curbing emissions.

Closer inspection of the functions $\Gamma^{\bar{t}}$ and $\Gamma^{\bar{s}}$ (Appendix) reveals the elasticity of substitution in consumption, $\sigma:=\frac{d(x / z) /(x / z)}{d\left(U_{z} / U_{x}\right) /\left(U_{x} / U_{z}\right)}$, as a determinant of relative policy effectiveness. More specifically, we find that $\left.\lim _{\sigma \rightarrow 0}\left|\Gamma_{e}^{\bar{t}}(e, \bar{t})\right|=\left|\Gamma_{e}^{\bar{s}}(e, \bar{s})\right|=\frac{p_{e} p_{z} z+\left(p_{e}+t\right) x}{\left(p_{e}+t\right)\left[\left(p_{z}+s\right) z+x\right]} \in\right] 0,1[$, $\lim _{\sigma \rightarrow \infty}\left|\Gamma_{e}^{\bar{t}}(e, \bar{t})\right|=\infty$ and $\lim _{\sigma \rightarrow \infty}\left|\Gamma_{e}^{\bar{s}}(e, \bar{s})\right|=0$. The implication is that the size of the wedge between the $s=0$ curve and the $t=0$ curve depends on the elasticity of substitution in consumption, $\sigma$. In the polar case $\sigma=0$ both commodities are strictly complementary, while they tend to become perfect substitutes if $\sigma \rightarrow \infty$. The less substitutable both commodities are in consumption the less divergent are the $s=0$ curve and the $t=0$ curve, i.e. the smaller is the area spanned by both curves to the left of their common point in Figure 2.

To better understand the role of $\sigma$, recall that the function of the equilibrium energy price, $p_{z}=P^{z}(s, t)$, has the properties $P_{t}^{z}>0$ and $P_{s}^{z}<0$ for intermediate values of $\sigma$. In case of $\sigma \rightarrow \infty$, both partial derivatives, $P_{t}^{z}$ and $P_{t}^{z}$, tend toward zero. Hence, the transmission channel closes and the consequence is that now the subsidy only promotes green energy and the tax only reduces emissions. Conversely, if $\sigma \rightarrow 0$, the $s=0$ curve and the $t=0$ curve tend to collapse into a single negatively sloped curve.

We summarize the above discussion in

\section{Result 3.}

(i) The set $\mathbb{Z}:=\left\{(e, g) \in \mathbb{R}_{+}^{2} \mid e=E(s, t), g=G(s, t),(s, t) \in \mathbb{R}_{+}^{2}\right\}$ of equilibrium allocations $(e, g)$ attainable through tax-subsidy policies consists of all points on the $\bar{s}=0$ curve, 
of all points on the $\bar{t}=0$ curve and of all points in the area above the $\bar{s}=0$ curve and below the $\bar{t}=0$ curve.

(ii) The interior of the set $\mathbb{Z}$ is non-empty for all $\sigma>0$. The direct effects of both the tax and the subsidy instrument are the stronger relative to their indirect effects, the greater is $\sigma$. For $\sigma \rightarrow 0$ both instruments tend to become equally effective in cutting emissions and promoting green energy.

Obviously, in Figure 2 the set of equilibrium allocations $(e, g)$ consists of the area $A D H$ including the boundary lines of $A D H$. It is not feasible to attain through tax-subsidy policies with positive rates any pair $(e, g)$ of emissions and green energy located below the $s=0$ curve $A D$ or above the $t=0$ curve $A H$. It is also worth pointing out that each point in the interior of the triangle $A D H$ is uniquely characterized by some pair of positive rates $(s, t)$. For example, if the regulator fixes the rates at $t=t_{1}$ and $s=s_{1}$, she will reach the point $M$ in Figure 2. For the sake of completeness we will briefly comment on the areas I, II and IV of Figure 2 later in Section 4.

\subsection{Incidence of tax-subsidy policies: further results}

In Section 3.1 above, we have restricted our attention to determining the levels of emissions and green energy resulting from variations in the tax and/or subsidy. We now complement this analysis by showing how variations in the rates $(\bar{s}, \bar{t})$ affect other equilibrium prices and quantities. We also introduce a fossil fuel price shock and determine the impact of this shock on the economy when the rates $(\bar{s}, \bar{t})$ remain unchanged. The comparative statics yield

Result 4. Suppose $(\bar{s}, \bar{t}) \in \mathbb{R}_{+}^{2}$ are the rates of the initial tax-subsidy policy and

- the regulator increases $\bar{s}$ while keeping $\bar{t}$ constant or

- the regulator increases $\bar{t}$ while keeping $\bar{s}$ constant or

- the fossil fuel price increases while the regulator maintains the rates $(\bar{s}, \bar{t})$.

(i) The resultant equilibrium displacement effects are as listed in Table 1.

\begin{tabular}{|c||c|c|c|c|c|c|}
\cline { 2 - 7 } \multicolumn{1}{l||}{} & $\hat{e}$ and $\hat{b}$ & $\hat{g}$ & $\hat{p}_{z}$ & $\hat{x}$ & $\hat{z}$ & $\hat{u}$ \\
\hline \hline$\hat{s}$ & - & + & - & $?$ & $-?$ & - \\
\hline$\hat{t}$ & - & + & + & $?$ & $-?$ & - \\
\hline$\hat{p}_{e}$ & - & $+?$ & $+?$ & $+?$ & $-?$ & $-?$ \\
\hline
\end{tabular}

Table 1: Incidence of variations in the subsidy, the tax and the price of fossil fuel ${ }^{11}$ (Notation: $\hat{x}:=d x / x$ for $x=s, t, p_{e}$ etc.)

\footnotetext{
${ }^{11}$ To keep focused we omit in Table 1 (and in the subsequent Table 2) the effects on $k_{g}, k_{x} p_{k}$ and $x_{s}$. For more information, we refer the reader to the Appendix.
} 
(ii) The plus and minus signs in Table 1 with question marks attached are valid under the following sufficient conditions:

(a) $\hat{z} / \hat{t}<0$, if $\alpha_{b}$ or $\sigma$ is sufficiently large;

(b) $\hat{z} / \hat{s}<0$, if $\alpha_{b}$ is sufficiently large and $\hat{z} / \hat{s}>0$, if $\sigma$ is sufficiently large.

(c) $\hat{p}_{z} / \hat{p}_{e}>0$, if $\left.\varepsilon_{e e}^{b} \in\right] 0,1\left[\right.$ and $x>p_{z} z$;

(d) $\hat{g} / \hat{p}_{e}>0$ and $\hat{u} / \hat{p}_{e}<0$, if $\left.\varepsilon_{e e}^{b} \in\right] 0,1\left[, x>p_{z} z\right.$ and $t \geq p_{e}$;

(e) $\hat{x} / \hat{p}_{e}>0$, if $\hat{p}_{z} / \hat{p}_{e}>0$ and $\sigma$ is sufficiently large;

(f) $\quad \hat{z} / \hat{p}_{e}<0$, if $\alpha_{b}$ is sufficiently large;

where $\left.\alpha_{b}:=\frac{b}{z} \in\right] 0,1\left[\right.$ and $\varepsilon_{e e}^{b}:=-\frac{e Q_{e e}^{b}}{Q_{e}^{b}}>0$ and $\sigma$ is defined in Result 2 .

Comments on Result 4. As far as exogenous tax or subsidy changes are concerned, Result 4 restates Result 1 in the first and second column of Table 1 . The third column shows, as expected, that an increase in the subsidy rate reduces - and an increase in the tax rate raises - the consumer price of energy ${ }^{12}$. This may tilt the political decision in favor of subsidizing green energy, if policy makers consider a low consumer price of energy as an important political target (Skytte 2006). The response to tax or subsidy hikes of total energy and good $X$ are not so clear. According to the Results 4iia and 4iib total energy consumption is the more likely to decline, the greater is the black energy share of total energy consumption, $\alpha_{b}$. Furthermore, since raising $t[s]$ makes energy more [less] expensive, the substitution effect away from energy consumption [toward more energy consumption] explains as to why total energy consumption declines following a tax increase $(\hat{z} / \hat{t}<0)$ [total energy consumption rises following a subsidy increase $(\hat{z} / \hat{s}>0)]$ for sufficiently large values of the substitution elasticity $\sigma$.

Result 4 also informs about the impacts of an exogenous increase in the price of fossil fuel under the assumption that the regulator sticks to the tax-subsidy policy that was in operation before the price increase. According to Table 1, the fossil fuel price shock reduces the use of fossil fuel and hence the production of black energy. These effects are clearly expected. Although the impact on other quantities and prices is not clear, in general, the Results 4iic through 4iif offer additional insights. The conditions $\left.\varepsilon_{e e}^{b} \in\right] 0,1\left[\right.$ and $x>p_{z} z$ which are needed to secure $\hat{p}_{z} / \hat{p}_{e}>0$ are not restrictive, because $\left.\varepsilon_{e e}^{b} \in\right] 0,1[$ is satisfied by all concave isoelastic production functions of the form $Q^{f}(e)=e^{\mu}$ with $\left.\mu \in\right] 0,1[$ and because there is clear empirical evidence that total expenditure of consumers on items other than energy is greater than expenditure on energy $\left(x>p_{z} z\right)$. In contrast, the additional condition $t \geq p_{e}$ needed in Result 4iid to secure $\hat{g} / \hat{p}_{e}>0$ appears to be rather strong. (Recall though, that we deal with sufficient conditions only). According to the Results 4iie and 4iif, a fossil fuel price

\footnotetext{
${ }^{12}$ Our result that the consumer price of energy shrinks when green energy is promoted confirms the result of Fisher and Newell (2008) and Fischer (2006) as well as empirical findings of Rathmann (2008) and Abrell and Weigt (2008). Other studies, e.g. Clemmer et al. (1999) or Palmer and Burtrow (2005), find that green energy promotion raises the energy price. However, their results are obtained in models where changes in the price and supply of fossil fuels are endogenous, which we have excluded by focusing on a small fossil-fuel importing country.
} 
hike expands the consumption of the consumer good and reduces total energy consumption ${ }^{13}$ under plausible conditions.

Consider finally the welfare impact of changes in the tax-subsidy policy. The message of Result 4 is that raising the rate of the tax or the subsidy, ceteris paribus, leads to a welfare loss, as expected. According to our comparative-static analysis (Result 4iid) an exogenous hike of the fossil fuel price reduces utility $\left(\hat{u} / \hat{p}_{e}<0\right)$ under implausible sufficient conditions only. Yet the following conclusive argument shows that $\hat{u} / \hat{p}_{e}<0$ holds unconditionally. For the black energy firm it makes no difference whether its fossil fuel costs rise via an increase in price or in tax. Insofar, the price hike works like a tax increase and such a tax increase leads to a welfare loss $(\hat{u} / \hat{t}<0)$ as shown in Table 1 . However, if we consider a tax increase, the incremental tax revenues are recycled in our model raising the consumer's income. In contrast, if we consider a fuel price increase instead, the import bill is higher than in case of the tax increase and needs to be paid by surrendering an extra amount of domestically produced consumption good to the fuel exporting country. From $\hat{u} / \hat{t}<0$ (see above) therefore follows $\hat{u} / \hat{p}_{e}<0$, a fortiori.

\section{Incidence of quantity-based mixed policies: cap and trade schemes combined with tradable green certificates schemes}

So far, our focus has been on tax-subsidy policies consisting of an emissions tax, $\bar{t}$, and/or a green subsidy, $\bar{s}$. We now turn to mixed quantity-based policies that presuppose the existence of some cap $\bar{e}$ for emissions and a standard $\bar{g}$ for green energy. More specifically, the regulator takes $\bar{e}$ as the upper limit for emissions, $e$, and $\bar{g}$ as the lower bound for green energy, $g$, i.e. she sees to it that the realized levels of emissions $e$ and/or $g$ satisfy $^{14}$

$$
e \leq \bar{e} \quad \text { and } \quad g \geq \bar{g} \text {. }
$$

The regulator issues the amount $\bar{e}$ of tradable emissions permits and allocates them to the firms in the black energy sector (via free allocation or auction). As for the standard $\bar{g}$, the regulator allows the firms in the green energy sector to issue a green certificate for each unit of green energy produced. The firms can then sell their certificates to the producers of black energy who, in turn, are obliged to purchase a certain number of green certificates, say $\theta$, per unit of emissions released. With the emissions cap $\bar{e}$ in operation, we clearly have ${ }^{15}$ $\theta=\bar{g} / \bar{e}$. To keep our notation simple, we continue to make use of the variables $(s, t)$ but now $t$ denotes the price on the market for emissions permits (permit price) and $s$ denotes the

\footnotetext{
${ }^{13}$ Obviously, this scenario implies an increase in the production of green energy.

${ }^{14}$ Since the standards are political goals, the inequalities in (20) are expected to be satisfied as equalities unless equality cannot be attained (see below).

${ }^{15}$ The complex institutional details of schemes of trading emissions and green certificates in practice are the subject of a large literature that is not the focus of the present paper. The specific institutional arrangements and variations of the relatively recent green certificates schemes are discussed e.g. in OECD/IEA (2008) and in Federal Environmental Agency (2007). It is easy to show that quantity-based policies employing taxes resp. subsidies on the one hand and regulation through tradable certificates schemes on the other hand are equivalent procedures for implementing the standards $\bar{e}$ and $\bar{g}$ at the level of abstraction of our present 'stylized' model. The tax rate $\tilde{t}$ prevailing in the tax-and-standard regime is equal to the equilibrium price in the market for emissions permits and the subsidy rate $\tilde{s}$ in the subsidy-and-standard regime is equal to the equilibrium price in the market for green certificates.
} 
price on the market for tradable green certificates (certificate price). In equilibrium there exists a pair of prices $(\tilde{s}, \tilde{t})$ such that ${ }^{16} G(\tilde{s}, \tilde{t}) \geq \bar{g}$ and $E(\tilde{s}, \tilde{t}) \leq \bar{e}$.

Result 5. Suppose the initial standards $(\bar{e}, \bar{g})$ satisfy $\bar{e}<E(0,0)$ and $\bar{g}>G(0,0)^{17}$, and

- $\quad$ the cap $\bar{e}$ is relaxed $(\hat{\bar{e}}>0)$ while keeping the standard $\bar{g}$ constant or

- $\quad$ the standard $\bar{g}$ is increased $(\hat{g}>0)$ while keeping the cap $\bar{e}$ constant or

- $\quad$ the fossil fuel price increases while the standards $(\bar{e}, \bar{g})$ remain unchanged.

The resultant equilibrium displacement effects are as listed in Table 2.

\begin{tabular}{|c||c|c|c|c|c|c|c|}
\cline { 2 - 7 } \multicolumn{1}{c|}{} & $\hat{b}$ & $\hat{t}$ & $\hat{s}$ & $\hat{p}_{z}$ & $\hat{x}$ & $\hat{z}$ & $\hat{u}$ \\
\hline \hline$\hat{g}$ & 0 & - & + & - & - & + & - \\
\hline$\hat{e}$ & + & - & + & - & - & + & + \\
\hline$\hat{p}_{e}$ & 0 & - & + & - & - & 0 & - \\
\hline
\end{tabular}

Table 2: Variations in policy parameters and in the fossil fuel price under the combined schemes of 'cap and trade' and 'tradable green certificates'

Comments on Result 5. When compared with Result 4 (Table 1) Table 2 reveals a dual relationship between variations in political parameters of price-based policies and the corresponding parameters of quantity-based policies. In formal terms the duality is between $\hat{g} /\left.\hat{s}\right|_{\hat{t}=0}>0$ (Table 1) and $\hat{s} /\left.\hat{g}\right|_{\hat{e}=0}>0$ (Table 2) as well as between $\hat{e} /\left.\hat{t}\right|_{\hat{s}=0}<0$ (Table 1) and $\hat{t} /\left.\hat{e}\right|_{\hat{g}=0}<0$ (Table 2). It tends to holds for the other endogenous variables as well but there are exceptions. For example, $\hat{z} /\left.\hat{s}\right|_{\hat{t}=0}$ may be negative (Result 4iib) while $\hat{z} /\left.\hat{g}\right|_{\hat{e}=0}$ is unambiguously positive (Table 2 ).

As the impact of changes in policy parameters on the consumer price of energy received much attention in both the political arena and in the energy literature, we wish to highlight the clearcut results of our analysis. In the tax-subsidy case, raising the tax increases the energy price and raising the subsidy lowers it (Table 1). The quantity-based mixed policy yields the dual results. Strengthening the emissions cap $(\hat{e}<0)$ increases the energy price, while a higher green standard $(\hat{g}>0)$ lowers it (Table 2). Our finding that the consumer price of energy increases upon raising the green standard $\left(\hat{p}_{z} / \hat{g}>0\right)$ contrasts with the result in the model of

\footnotetext{
${ }^{16}$ To be precise, $(\tilde{s}, \tilde{t})$ must also satisfy the following condition: There is no other policy $\left(s^{\prime}, t^{\prime}\right)$ satisfying $R\left(s^{\prime}, t^{\prime}\right) \geq r_{c}, B\left(s^{\prime}, t^{\prime}\right) \leq b_{c}$ as well as $R\left(s^{\prime}, t^{\prime}\right) \leq R(\tilde{s}, \tilde{t})$ and $B\left(s^{\prime}, t^{\prime}\right) \geq B(\tilde{s}, \tilde{t})$, where a strict inequality sign holds in at least one of the last two inequalities.

${ }^{17}$ See Footnote 22.
} 
Jensen and Skytte (2003, p. 67) who find that introducing a green quota leads to an ambiguous change in the consumer energy price.

Consider now an increase in the fossil fuel price $\left(\hat{p}_{e}>0\right)$. Since the production of green and black energy is fixed under the policy $(\bar{e}, \bar{g})$, such an increase cannot obviously affect energy production at all. The black energy firm reduced its demand for fossil fuel, ceteris paribus. Hence the permit price needs to decline thus encouraging the black energy firm to keep up its production while the certificate price goes up to avoid the expansion of green energy. The effect on the energy price is negative $\left(\hat{p}_{z} /\left.\hat{p}_{e}\right|_{\hat{g}=0, \hat{e}=0}<0\right)$ because the demand for energy declines while the supply is constant by presupposition. That result is counterintuitive because at first glance one would expect the cost increase $\left(\hat{p}_{e}>0\right)$ being passed on to the consumers by raising the energy price $p_{z}$.

The result $\hat{p}_{z} /\left.\hat{p}_{e}\right|_{\hat{g}=0, \hat{e}=0}<0$ contrasts with Result 4iic where we found that $\hat{p}_{z} /\left.\hat{p}_{e}\right|_{\hat{t}=0, \hat{s}=0}>0$ under the conditions $\left.\varepsilon_{e e}^{b} \in\right] 0,1\left[\right.$ and $x>p_{z} z$. To see the reason for the difference in sign note that Table 1 also contains the result $\hat{p}_{z} /\left.\hat{t}\right|_{\hat{p}_{e}=0, \hat{s}=0}>0$. For the black energy firm the cost effects of the changes $\left(\hat{t}>0, \hat{p}_{e}=0\right)$ and $\left(\hat{t}=0, \hat{p}_{e}>0\right)$ are the same. We also know that $\hat{e} /\left.\hat{p}_{e}\right|_{\hat{t}=0, \hat{s}=0}<0$ (Table 2) as well as $\hat{e} /\left.\hat{t}\right|_{\hat{p}_{e}=0, \hat{s}=0}<0$ (Table 1) which is compatible with negative $\hat{p}_{z}$ and with positive (but sufficiently small) $\hat{p}_{z}$. If the production function for black energy exhibits little curvature, $\left.\varepsilon_{e e}^{b} \in\right] 0,1[$, then one would see a sharp decline in black energy, if $\hat{t}>0, \hat{p}_{e}=0$ and (!) $\hat{p}_{z}=0$. To push back the resultant increase in energy demand and to reduce (but not overcompensate) thereby the decline in black energy an increase in the price $p_{z}$ is required. The reason why $\hat{p}_{z} /\left.\hat{t}\right|_{\hat{p}_{e}=0, \hat{s}=0}>0$ holds unconditionally whereas $\hat{p}_{z} /\left.\hat{p}_{e}\right|_{\hat{t}=0, \hat{s}=0}>0$ holds under some qualifications only is that in the latter scenario we have an income loss owing to the fact that the import bill is larger than in the former scenario.

According to Table 2, the variations $\hat{g}>0, \hat{e}<0$ and $\hat{p}_{e}>0$ all lead to welfare losses. The welfare loss following an increase in the fossil fuel price $\left(\hat{u} / \hat{p}_{e}<0\right)$ is particularly straightforward. Consumers are forced to consume as much energy as before the fuel price shock while the consumption of good $X$ declines because a larger share of the consumption good produced is needed to pay for the (higher) import bill.

The welfare impact of mixed quantity-based policies can be conveniently illustrated and interpreted in Figure 3. The lines AE and AG in Figure 3 are the same as the lines AD and AH, respectively, in Figure 2. Maximum welfare is attained in the absence of any regulation, i.e. in point A. Straightforward implications of the Results 4 and 5 are welfare-indifference curves such as $\bar{e}_{B} B N u_{B}, \bar{e}_{C} C M u_{C}, \bar{e}_{D} D L u_{D}$ etc. in Figure 3 that are ranked in the order of diminishing welfare, i.e. $u_{A}>u_{B}>u_{C}>u_{D}$ etc. Assuming that welfare is measureable, we are able to specify the welfare costs of quantity-based policies. Consider for example some mixed policy 
$(\bar{e}, \bar{g})$ with $(\bar{e}, \bar{g})$ being a point in the area II of Figure $2,{ }^{18}$ such as the policy $\left(\bar{e}_{C}, \bar{g}_{Q}\right)$ in Figure 3. The total welfare costs of that policy are equal to the difference $u_{D}-u_{A}>0$. However, owing to the interaction of curbing emissions and promoting green energy it is not possible to assign to individual standards an unambiguous welfare loss. To see that, suppose first that an emissions cap already exists and is to be complemented by a green standard. In our example, the costs of implementing the emissions cap $\bar{e}_{C}$ alone are $u_{C}-u_{A}$. These costs are positive but less than $u_{D}-u_{A}$. The costs of adding the green standard $\bar{g}_{Q}$ to the emissions cap $\bar{e}_{C}$ are $u_{D}-u_{C}$. Alternatively, suppose the green standard $\bar{g}_{Q}$ preexists and is to be complemented by the emissions cap $\bar{e}_{C}$. In that case the costs of implementing the green standard alone are $u_{B}-u_{A}$ and the costs of adding the emissions cap $\bar{e}_{C}$ to the green standard $\bar{g}_{Q}$ are $u_{D}-u_{B}$. As a consequence ${ }^{19}$, all we can say on welfare losses is that for the mixed policy $\left(\bar{e}_{C}, \bar{g}_{Q}\right)$ to be net welfare improving it is necessary that its total external social benefits exceed $u_{D}-u_{A}$.

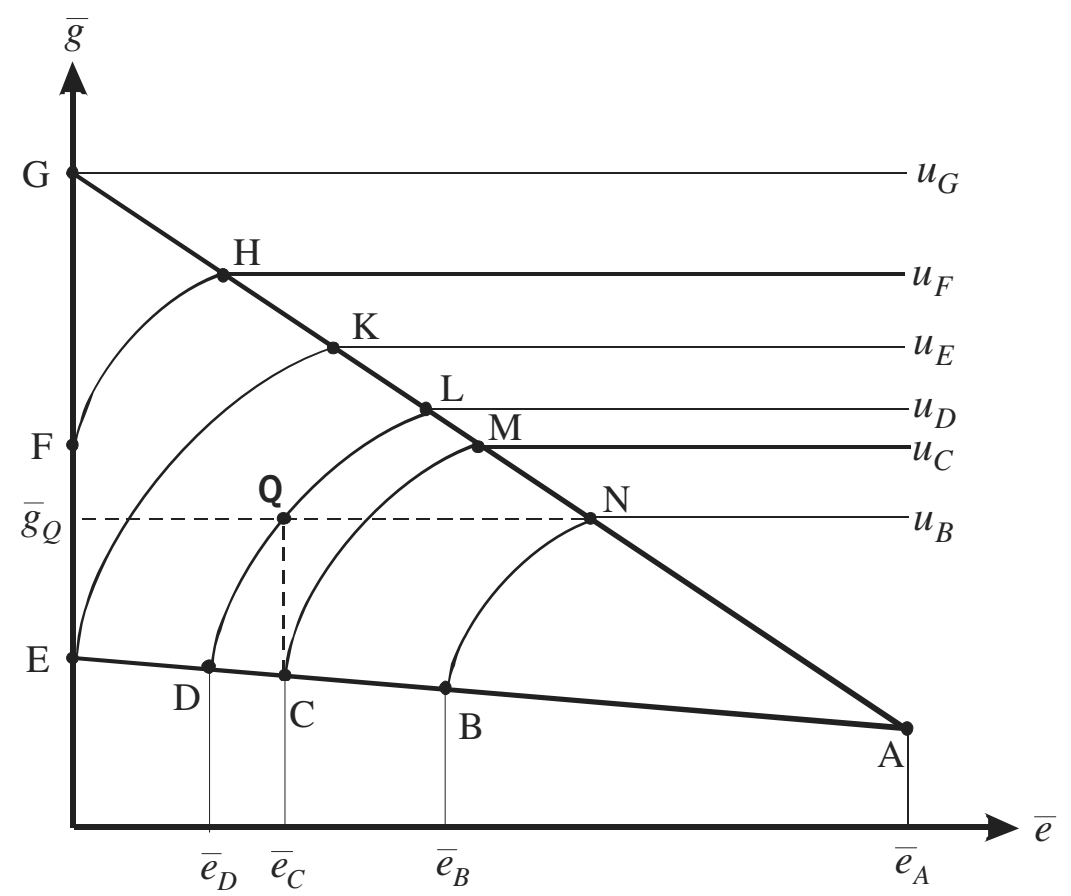

Figure 3: Mixed quantity-based policies and welfare

Suppose the gross social benefits of curbing emissions to the level $\bar{e}_{C}$ exceed $u_{C}-u_{A}$ but adding the green standard $\bar{g}_{Q}$ does not add any benefits. In that case, cost effectiveness would

\footnotetext{
${ }^{18}$ In formal language, the mixed policy in area II of Figure 2 is a policy $(\bar{e}, \bar{g}) \in \operatorname{int} A$ with the set $A$ as defined in Result 3.

${ }^{19}$ Recall that we have not included the potential social benefits of mixed policies in our formal model. Therefore, we cannot apply the cost-benefit criterion to determine whether a mixed policy such as $\left(\bar{e}_{C}, \bar{g}_{Q}\right)$ enhances net welfare.
} 
clearly require refraining from implementing any binding green standard. Recall, however, that it is not the focus of the present paper to deal with the existence or non-existence of such benefits. We rather contend ourselves with the following observations. If it is true that expanding green energy comes without intrinsic benefits other than its emissions reducing side effect $^{20}$, demands for abolishing green energy support schemes are valid ${ }^{21}$. In analogy, curbing emissions would be wasteful as well, if supporting green energy would yield no social benefits. According to Result 3ii the welfare losses from applying the 'wrong' policy are the smaller, the smaller is the elasticity of substitution in demand (and hence the smaller is the direct price elasticity of energy demand).

In our preceding analysis, we explored the incidence of variations in policy parameters focusing on one parameter change at a time. In December 2008 the EU Parliament agreed on a package targeting 20\% reductions in greenhouse gas emissions and a $20 \%$ share of renewables in overall EU energy consumption (and 20\% increase in energy efficiency, which we will neglect here) by 2020 . That gives rise to the question as to what the effects are in our framework of analysis of a simultaneous change in the quantitative standards $\bar{e}$ and $\bar{g}$. The answer is straightforward from invoking the Figures 2 and 3. If the initial situation is given be a point such as $M$ in Figure 2 or $Q$ in Figure 3 the EU targets correspond to moving to a point northwest of $M$ and $Q$, respectively. Figure 3 gives us the welfare costs of that policy and Figure 2 shows the implied changes in the rates of the tax and the subsidy. Suppose the target point (not marked in Figure 2) lies in the interior of the area $L K G M$ in Figure 2. If it lies above the line $M K$, the tax will fall and the subsidy will rise; if it lies below MK, the tax rate will fall and the subsidy will rise. The actual position of the target point is an empirical issue depending on technologies and on the elasticity of substitution in demand.

Up to now, we have restricted our analysis to the comparative statics of binding pairs of standards $(\bar{e}, \bar{g})$. The set of such pairs of standards is characterized in Result 3 and corresponds to the area II (triangle $D H A$ ) in Figure 2. The area IV in Figure 2 is irrelevant but if $(\bar{e}, \bar{g})$ is in area I [in area III] the green standard [the emissions cap] is not binding. The implications of non-binding standards are conveniently illustrated in the Figures $4 \mathrm{a}$ and $4 \mathrm{~b}$ that also display the comparative statics of variations in the green standard (Figure 4a) and the emissions cap (Figure $4 \mathrm{~b}$ ). Figure $4 \mathrm{a}$ shows the equilibrium values of the endogenous variables $t, s, p_{z}$ and $u$ corresponding to successive increases of the green standard $\bar{g}$. That is, Figure 4 a is generated by moving along a straight line parallel to the ordinate in Figure 2. For example, take the line starting in $e_{C}$, passing through $C, M, G$ and reaching beyond $G$. Likewise, Figure $4 \mathrm{~b}$ is generated by moving along a straight line parallel to the abscissa in Figure 2 such as the line starting in $g_{C}$, passing through $C, E, N$ and reaching beyond $N$.

To see the straightforward policy implications of Figure 4, suppose the country wants to secure a pair of standards $(\bar{e}, \bar{g})$ that is to be implemented by an emissions tax and a green subsidy. We need to distinguish three cases:

\footnotetext{
${ }^{20}$ The emissions reducing side effect (Section 3) of supporting green energy fails to take effect in the presence of a binding emissions cap. In that case it is 'transformed' into a permit price reducing effect.

${ }^{21}$ Based on the (correct) argument that, if an emissions cap is in operation, the level of emissions is unaffected by the green subsidy (see previous footnote) the German scientific council at the Federal Ministry of the Economy (Wissenschaftlicher Beirat beim Bundesministerium für Wirtschaft und Arbeit 2004) suggested abolishing the German Renewable Energy Act.
} 


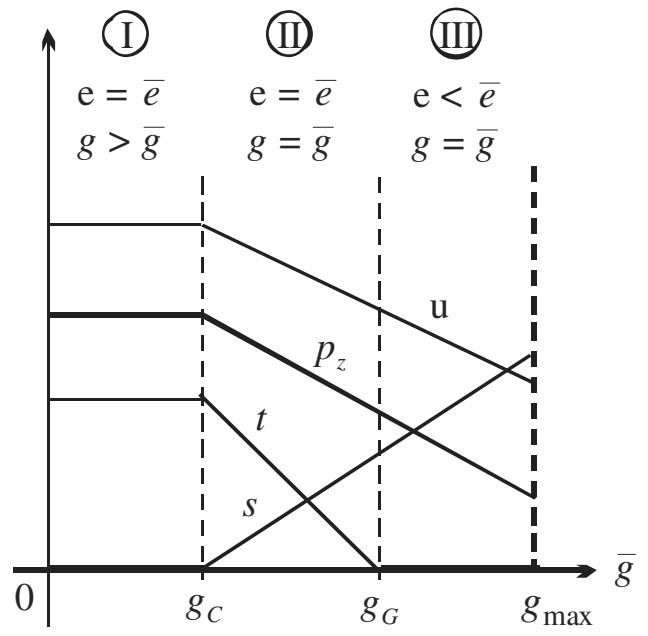

Figure 4a

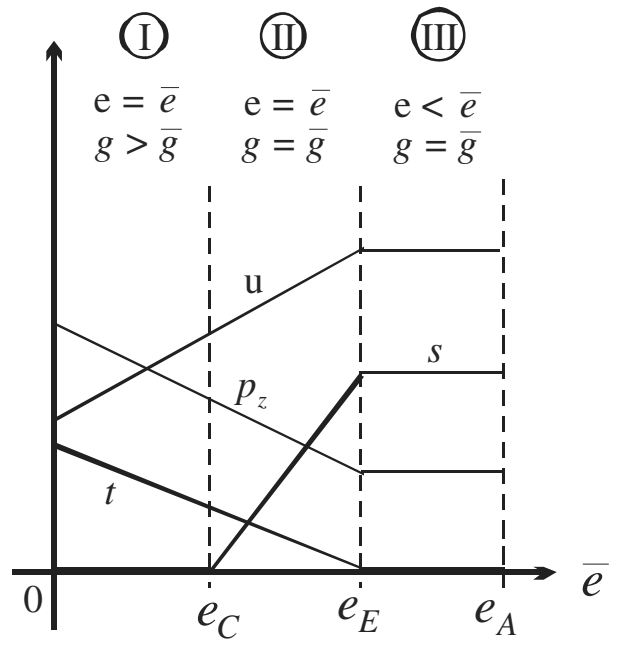

Figure $4 b$

Figure 4: Impacts of successive changes in either the green standard (Figure 4a) or the emissions cap (Figure $4 b$ )

(a) $(\bar{e}, \bar{g})$ is in area I of Figure 4 (and Figure 2). In that case, the tax alone does the job because the standard $\bar{g}$ is not binding. Without any promotion of green energy and a suitable tax policy the allocation $\left[e=E(s=0, t)=\bar{e}, g=\Gamma^{\bar{s}}(\bar{e}, s=0)>\bar{g}\right]$ will be attained.

(b) $(\bar{e}, \bar{g})$ is in area II of Figure 4. Now a mixed tax-subsidy policy is needed, and there is a unique pair of positive rates $(s, t)$ that implements $(\bar{e}, \bar{g})$.

(c) $(\bar{e}, \bar{g})$ is in area III of Figure 4. In that case, the subsidy alone does the job because the emissions cap $\bar{e}$ is not binding. Without any tax to curb emissions and a suitable subsidy for green energy, the allocation $\left[e=E(s, t=0)<\bar{e}, g=\Gamma^{\bar{s}}(e, s)=\bar{g}\right]$ will be attained.

It is obvious that the case (b) is the only relevant scenario for practical policy.

\section{Concluding remarks}

In a general equilibrium model of a small fossil-fuel importing economy, we have studied the incidence of price-based and quantity-based mixed policies each consisting of two policy instruments (or schemes) one of which targets the promotion of green energy and the other the reduction of carbon emissions. We have investigated the impact of policy changes on the allocation, on prices, on welfare as well as the effects of fossil-fuel price shocks under those policy regimes. Policy instruments directed toward promoting green energy are shown to have the side effect of reducing carbon emissions and vice versa but the direct effects are stronger than the side effects, the more so, the greater is the elasticity of substitution in consumption between energy and consumption goods. Hence, we identify the substitution elasticity as an important determinant of the individual instruments' relative effectiveness with regard to both targets. A straightforward implication of the difference in relative effectiveness is that if the country only wants to reduce emissions - or only wants to promote green energy - it must not use a mixed policy but rather a single instrument, namely that particular instrument which 
addresses the target directly. Otherwise, the policy would not be cost-effective. We also show that if the country pursues both the reduction of emissions and the promotion of green energy, a mixed (quantity-based or price-based) policy is needed, if and only if both constraints are binding.

We calculate the welfare costs of policy changes but do not explore conditions for optimal mixed policies, which would have required considering (potential) external social costs in the formal model. The largely undisputed rationale for efforts to reduce emissions is, of course, the global negative externality of climate change. The rationale for promoting the (domestic) production of green energy is less clear. Economists use to reject regulatory action unless significant externalities or market failures are shown to exist. The discussion in the literature about various kinds of market imperfections in the context of green energy (Gonzales 20067) is controversial with some studies recommending and others rejecting as valid suggested reasons for policies promoting green energy. Nonetheless, as pointed out in the introduction, many countries and the EU have committed to and have legislated domestic support of green energy. Among the various reasons given, the argument to improve security of energy supply through reducing the dependence on imports of fossil fuels is prominent. While that argument is plausible, we are not aware of studies demonstrating rigorously that the way the market system copes with that challenge is unsatisfactory.

There is some literature taking low energy prices as a political target in its own right ${ }^{22}$. Given our result that the promotion of green energy will lower the energy price while cutting emissions will increase it, proponents of low energy prices might suggest to complement an emissions reduction policy with green energy promotion in order to prevent the energy price from rising. It is possible within the framework of our model to calculate the (welfare) costs of keeping the energy price constant through adopting a specific mix of green energy promotion and carbon emissions reduction. One would then need to explore whether that is a costeffective way to prevent energy prices from rising.

\section{References}

Abrell J, Weigt H (2008) The interaction of emissions trading and renewable energy promotion. WP-EGW-06, Dresden University of Technology

Baumol WJ, Oates WE (1971) The use of standards and prices for the protection of the environment. Swedish Journal of Economics 73:42-54

Bode S, Groscurth H (2006) The effect of the German renewable energy act on the electricity price. HWWA Discussion Paper 358

Bovenberg AL, de Moij RA (1994) Environmental levies and distortionary taxes. American Economic Review 84:1085-1089

Federal Government of Germany/Bundesregierung (2008) Gesetz zur Förderung erneuerbarer Energien im Wärmebereich. Bundesgesetzblatt Jg. 2008 Teil I Nr. 36 vom 18. 8. 2008,

Bye T, Bruvoll A (2008) Multiple instruments to change energy behaviour: The emperor's new clothes? Energy Efficiency 1:373-386

Clemmer SL, Nogee A, Brower MC, Jefferiss P (1999) A powerful opportunity: Making Renewable electricity the standard. Union of Concerned Scientists, Cambridge

\footnotetext{
${ }^{22}$ See Bode and Groscurth (2006), Fischer (2006) and Rathmann (2007) for an analysis of the effects of green energy promotion on energy prices.
} 
COM (2007) Communication from the Commission to the European Council and the European Parliament of 10 January 2007 "An energy policy for Europe". http://europa.eu/legislation_summaries/energy/european_energy_policy/27067_en.htm

European Commission Directorate-General Environment (2005) Interactions of the EU ETS with green and white certificate schemes. Green final report. Studies on Economic Aspects of Climate Change http://ec.europa.eu/environment/climat/studies.htm

Federal Environmental Agency (Umweltbundesamt) (2007) Monitoring and evaluation of policy instruments to support renewable electricity in EU member states. Final report http://www.umweltbundesamt.de

Fischer C, Newell, RG (2008) Environmental and technology policies for climate mitigation Journal of environmental economics and management 55:142 - 162

Fischer C (2006) How can renewable portfolio standards lower electricity prices? RFF Discussion Paper 06-20-REV, Washington DC: Resources for the future

Gonzáles P del Río (2007) The interaction between emissions trading and renewable electricity support schemes. An overview over the literature. Mitigation and Adaptation Strategies for Global Change 12:1363-1390

Jensen SG, Skytte K (2003) Simultaneous attainment of energy goals by means of green certificates and emission permits. Energy Policy 31:63-71

Jensen SG, Skytte K (2004) Interaction between green support mechanisms at the European power market. Working Paper

Linares P, Santos FJ, Ventosa M (2008) Coordination of carbon reduction and renewable energy support policies. Climate Policy 8:377-394

OECD/IEA (2008) Deploying Renewables. Principles for Effective Policies. Paris

Palmer K, Burtraw D (2005) Cost-effectiveness of renewable electricity policies. Energy Economics 27:873 - 894

Rathmann M (2007) Do support systems for RES-E reduce EU-ETS-driven electricity prices. Energy Policy 35:342-349

Skytte K (2006) Interplay between environmental regulation and power markets. EUI Working Paper, RSCAS No. 2006/04, European University Institute

Sorrell S, Sijm J (2003) Carbon trading in the policy mix. Oxford Review of Economic Policy 19:420-437

Wissenschaftlicher Beirat beim Bundesministerium für Wirtschaft und Arbeit (2004) Zur Förderung erneuerbarer Energien.

\section{Appendix}

\section{Proof of Result 4 (excluding welfare changes)}

We assume small exogenous changes in the policy parameters $s$ and $t$ and in the fossil fuel price $p_{e}$. To determine the resulting changes in the new equilibrium we totally differentiate the equations (1) through (10) making use of the so-called hat calculus ( $\hat{x}:=d x / x$ etc.). 


$$
\begin{aligned}
& \varepsilon_{k}^{x} \hat{k}_{x}-\hat{x}_{s}=0 \\
& \text { with } \left.\varepsilon_{k}^{x}:=\frac{k_{x} Q_{k}^{x}}{x_{s}} \in\right] 0,1[ \\
& \left.\alpha_{b} \hat{b}+\left(1-\alpha_{b}\right) \hat{g}-\hat{z}=0 \quad \text { with } \alpha_{b}:=\frac{b}{z} \in\right] 0,1[ \\
& \varepsilon_{e}^{b} \hat{e}-\hat{b}=0 \\
& \text { with } \left.\varepsilon_{e}^{b}:=\frac{e Q_{e}^{b}}{b} \in\right] 0,1[ \\
& \varepsilon_{k}^{g} \hat{k}_{g}-\hat{g}=0 \\
& \text { with } \left.\varepsilon_{k}^{g}:=\frac{k_{g} Q_{k}^{g}}{g} \in\right] 0,1[ \\
& \alpha_{g} \hat{k}_{g}+\left(1-\alpha_{g}\right) \hat{k}_{x}=0 \\
& \text { with } \left.\alpha_{g}:=\frac{k_{g}}{k} \in\right] 0,1[ \\
& \hat{e}+\alpha_{x} \hat{x}-\left(1+\alpha_{x}\right) \hat{x}_{s}+\hat{p}_{e}=0 \\
& \text { with } \alpha_{x}:=\frac{x}{p_{e} e}>0 \\
& \sigma \hat{p}_{z}-\hat{x}+\hat{z}=0 \\
& \text { with } \sigma:=\frac{d(x / z) /(x / z)}{d\left(U_{z} / U_{x}\right) /\left(U_{z} / U_{x}\right)} \\
& \left.-\varepsilon_{e e}^{b} \hat{e}+\hat{p}_{z}-\alpha_{t} \hat{t}-\left(1-\alpha_{t}\right) \hat{p}_{e}=0 \quad \text { with } \quad \varepsilon_{e e}^{b}:=-\frac{e Q_{e e}^{b}}{Q_{e}^{b}}>0 \quad \text { and } \quad \alpha_{t}:=\frac{t}{p_{e}+t} \in\right] 0,1[ \\
& \left.-\varepsilon_{k k}^{g} \hat{k}_{g}-\hat{p}_{k}+\left(1-\alpha_{s}\right) \hat{p}_{z}+\alpha_{s} \hat{s}=0 \quad \text { with } \quad \varepsilon_{k k}^{g}:=-\frac{k_{g} Q_{k k}^{g}}{Q_{k}^{g}}>0 \quad \alpha_{s}:=\frac{s}{p_{z}+s} \in\right] 0,1[ \\
& \varepsilon_{k k}^{x} \hat{k}_{x}+\hat{p}_{k}=0 \\
& \text { with } \varepsilon_{k k}^{x}:=-\frac{k_{x} Q_{k k}^{x}}{Q_{k}^{x}}>0
\end{aligned}
$$

Starting from the system $\left(1^{\prime}\right)-\left(10^{\prime}\right)$ we derive the effect of changes in the parameters $p_{e}, s$ and $t$ on the endogenous variables.

(a): Consider $\hat{b}$ from (3'), $\hat{r}$ from (4') and $\hat{z}$ from (7') to turn equation (2') into

$$
\alpha_{f} \varepsilon_{e}^{f} \hat{e}+\left(1-\alpha_{f}\right) \varepsilon_{k}^{g} \hat{k}_{g}-\hat{x}+\sigma \hat{p}_{z}=0
$$

(b): Consider $\hat{k}_{x}$ from (5') and $\hat{p}_{k}$ from $\left(10^{\prime}\right)$ to turn (9') into

$$
-\varepsilon \hat{k}_{g}+\left(1-\alpha_{s}\right) \hat{p}_{z}=-\alpha_{s} \hat{s} \quad \text { with } \varepsilon:=\frac{\alpha_{g} \varepsilon_{k k}^{x}}{1-\alpha_{g}}+\varepsilon_{k k}^{g}>0
$$

(c): Consider $\hat{x}_{s}$ from (1') and $\hat{k}_{x}$ from (5') in (6') to obtain

$$
\frac{\alpha_{g}\left(1+\alpha_{x}\right) \varepsilon_{k}^{x}}{1-\alpha_{g}} \hat{k}_{g}+\alpha_{x} \hat{x}+\hat{e}=-\hat{p}_{e}
$$

(d): Consider $\hat{x}$ from (A1) to turn (A3) into

$$
\beta_{e} \hat{e}+\beta_{k} \hat{k}_{g}+\alpha_{x} \sigma \hat{p}_{z}=-\hat{p}_{e},
$$


where $\beta_{e}:=1+\alpha_{b} \alpha_{x} \varepsilon_{e}^{b}>0$ and $\beta_{k}:=\frac{\alpha_{g}\left(1+\alpha_{x}\right) \varepsilon_{k}^{x}}{1-\alpha_{g}}+\alpha_{x}\left(1-\alpha_{b}\right) \varepsilon_{k}^{g}>0$.

(e): We thus have reduced the system of equations $\left(1^{\prime}\right)-\left(10^{\prime}\right)$ to the system of the three equations ( $\left.8^{\prime}\right),(\mathrm{A} 2)$ and (A4) which we rewrite in the form:

$$
\left[\begin{array}{ccc}
\beta_{e} & \beta_{k} & \alpha_{x} \sigma \\
-\varepsilon_{e e}^{b} & 0 & 1 \\
0 & -\varepsilon & 1-\alpha_{s}
\end{array}\right] \cdot\left[\begin{array}{c}
\hat{e} \\
\hat{k}_{g} \\
\hat{p}_{z}
\end{array}\right]=\left[\begin{array}{c}
-\hat{p}_{e} \\
\alpha_{t} \hat{t}+\left(1-\alpha_{t}\right) \hat{p}_{e} \\
-\alpha_{s} \hat{s}^{2}
\end{array}\right] .
$$

The determinant of the matrix in (A5) is

$$
D:=\beta_{D} \varepsilon_{e e}^{b}+\beta_{e} \varepsilon>0, \quad \text { where } \beta_{D}:=\alpha_{x} \varepsilon \sigma+\left(1-\alpha_{s}\right) \beta_{k} .
$$

Applying Cramer's rule we solve (A5) for $\hat{e}, \hat{k}_{g}$ and $\hat{p}_{z}$ as follows:

$$
\begin{aligned}
& D \hat{e}=-\alpha_{t} \beta_{D} \hat{t}-\alpha_{s} \beta_{k} \hat{s}-\left(\beta_{D}+\varepsilon\right) \hat{p}_{e}, \\
& D \hat{k}_{g}=\alpha_{t}\left(1-\alpha_{s}\right) \beta_{e} \hat{t}+\alpha_{s}\left(\alpha_{x} \varepsilon_{e e}^{b} \sigma+\beta_{e}\right) \hat{s}+\left(1-\alpha_{s}\right)\left(\alpha_{t} \beta_{e}-\varepsilon_{e e}^{b}\right) \hat{p}_{e}, \\
& D \hat{p}_{z}=\alpha_{t} \beta_{e} \varepsilon \hat{t}-\alpha_{s} \beta_{k} \varepsilon_{e e}^{b} \hat{s}+\varepsilon\left[\left(1-\alpha_{t}\right) \beta_{e}-\varepsilon_{e e}^{b}\right] \hat{p}_{e} .
\end{aligned}
$$

(f): From the equations (1'), (3') and (4') it is straightforward that for $\hat{y}=\hat{s}, \hat{t}, \hat{p}_{e}$ $\operatorname{sgn}\left(\hat{x}_{s} / \hat{y}\right)=\operatorname{sgn}\left(\hat{k}_{x} / \hat{y}\right), \operatorname{sgn}(\hat{f} / \hat{y})=\operatorname{sgn}(\hat{e} / \hat{y})$ and $\operatorname{sgn}(\hat{g} / \hat{y})=\operatorname{sgn}\left(\hat{k}_{g} / \hat{y}\right)$. Likewise, $\left(5^{\prime}\right)$ and $\left(10^{\prime}\right)$ yield $\operatorname{sgn}\left(\hat{k}_{x} / \hat{y}\right)=-\operatorname{sgn}\left(\hat{k}_{g} / \hat{y}\right)$ as well as $\operatorname{sgn}\left(\hat{p}_{k} / \hat{y}\right)=-\operatorname{sgn}\left(\hat{k}_{x} / \hat{y}\right)=\operatorname{sgn}\left(\hat{k}_{g} / \hat{y}\right)$.

(g): According to (2'), (3') and (4'), the change in total energy consumption is $\hat{z}=\alpha_{f} \varepsilon_{e}^{f} \hat{e}+\left(1-\alpha_{f}\right) \varepsilon_{k}^{g} \hat{k}_{g}$. We combine this equation with (A6) and (A7), to obtain, after some rearrangement of terms,

$$
\begin{aligned}
D \hat{z}= & \alpha_{t}\left[\left(1-\alpha_{b}\right)\left(1-\alpha_{s}\right) \beta_{e} \varepsilon_{k}^{g}-\alpha_{b} \beta_{D} \varepsilon_{e}^{b}\right] \hat{t}+\alpha_{s}\left[\left(1-\alpha_{b}\right)\left(\alpha_{x} \varepsilon_{e e}^{b} \sigma+\beta_{e}\right) \varepsilon_{k}^{g}-\alpha_{b} \beta_{k} \varepsilon_{e}^{b}\right] \hat{s}+ \\
& +\left[\left(1-\alpha_{b}\right)\left(1-\alpha_{s}\right)\left(\alpha_{t} \beta_{e}-\varepsilon_{e e}^{b}\right) \varepsilon_{k}^{g}-\alpha_{f}\left(\beta_{D}+\varepsilon\right) \varepsilon_{e}^{b}\right] \hat{p}_{e} .
\end{aligned}
$$

(A9) shows that $\operatorname{sgn}(\hat{z} / \hat{y})$ is ambiguous for $\hat{y}=\hat{s}, \hat{t}, \hat{p}_{e}$. With $\hat{x}=\hat{z}+\sigma \hat{p}_{z}$ from (7'), it follows immediately that $\operatorname{sgn}(\hat{x} / \hat{y})$ is ambiguous for $\hat{y}=\hat{s}, \hat{t}, \hat{p}_{e}$ as well.

From (A8) we infer

$$
\frac{\hat{p}_{z}}{\hat{p}_{e}} \gtreqless 0 \Leftrightarrow\left(1-\alpha_{t}\right) \beta_{e} \gtreqless \varepsilon_{e e}^{b} .
$$

Invoking (8) and the definitions of $\alpha_{t}, \beta_{e}, \alpha_{b}, \alpha_{x}$ and $\varepsilon_{e}^{b}$ we find that

$$
\left(1-\alpha_{t}\right) \beta_{e}=\frac{p_{e}}{p_{e}+t}\left(1+\frac{x Q_{e}^{b}}{p_{e} z}\right)=\frac{p_{e}}{p_{e}+t}+\frac{p_{e}}{p_{e}+t} \cdot \frac{\left(p_{e}+t\right) x}{p_{e} p_{z} z}=\frac{p_{e}}{p_{e}+t}+\frac{x}{p_{z} z} .
$$

It follows that $\hat{p}_{z} / \hat{p}_{e}>0$, if $\left.\varepsilon_{e e}^{b} \in\right] 0,1\left[\right.$ and $x>p_{z} z$. Consider next (A7) and (4') to obtain 


$$
\frac{\hat{k}_{g}}{\hat{p}_{e}}=\frac{\hat{g}}{\varepsilon_{k}^{g} \hat{p}_{e}}=\left(1-\alpha_{s}\right)\left(\alpha_{t} \beta_{e}-\varepsilon_{e e}^{b}\right)=\left(1-\alpha_{s}\right)\left[\frac{t}{p_{e}}\left(1-\alpha_{t}\right) \beta_{e}-\varepsilon_{e e}^{b}\right] \text {. }
$$

Using (A10) we get $\frac{t}{p_{e}}\left(1-\alpha_{t}\right) \beta_{e}=\frac{t}{p_{e}+t}+\frac{x t}{p_{e} p_{z} z}$. A sufficient condition for $\hat{k}_{g} / \hat{p}_{e}>0$ and $\hat{g} / \hat{p}_{e}>0$ is therefore " $\left.\varepsilon_{e e}^{b} \in\right] 0,1\left[, x>p_{z} z\right.$ and $t \geq p_{e} "$.

\section{Proof of Result 2}

Both functions $\Gamma^{\bar{t}}$ and $\Gamma^{\bar{s}}$ have the point $[E(\bar{s}, \bar{t}), G(\bar{s}, \bar{t})]$ in common because $\Gamma^{\bar{s}}(e, \bar{s})=\Gamma^{\bar{t}}(e, \bar{t})=G(\bar{s}, \bar{t})$ for $e=E(\bar{s}, \bar{t})$. Since $\Gamma_{e}^{\bar{s}}=G_{t} \tilde{E}_{e}^{t}=G_{t} / E_{t}<0$ and $\Gamma_{e}^{\bar{t}}=$ $G_{s} \tilde{E}_{e}^{s}=G_{s} / E_{s}<0$, both functions are decreasing in $e$.

\section{Proof of Result 3ii}

We take the derivatives of the functions $\Gamma^{\bar{t}}$ and $\Gamma^{\bar{s}}, \Gamma_{e}^{\bar{t}}=G_{s} / E_{s}$ and $\Gamma_{e}^{\bar{s}}=G_{t} / E_{t}$, and invoke the equations (3'), (4'), (A6) and (A7) from the Appendix to obtain

$$
\Gamma_{e}^{\bar{s}}(e, \bar{s})-\Gamma_{e}^{\bar{t}}(e, \bar{t})=\frac{G_{s}}{\left|E_{s}\right|}-\frac{G_{t}}{\left|E_{t}\right|}=\frac{\left(\alpha_{x} \varepsilon_{e e}^{b} \sigma+\beta_{e}\right) \varepsilon_{k}^{g} g}{\beta_{k} \varepsilon_{e}^{b} b}-\frac{\left(1-\alpha_{s}\right) \beta_{e} \varepsilon_{k}^{g} g}{\beta_{D} \varepsilon_{e}^{b} b} .
$$

In view of the definition of $\beta_{D}$ it is straightforward that $\lim _{\sigma \rightarrow \infty}\left|\Gamma_{e}^{\bar{t}}(e, \bar{t})\right|=\infty$ and $\lim _{\sigma \rightarrow \infty}\left|\Gamma_{e}^{\bar{s}}(e, \bar{s})\right|=0$. Moreover, $\lim _{\sigma \rightarrow 0}\left|\Gamma_{e}^{\bar{t}}(e, \bar{t})\right|=\left|\Gamma_{e}^{\bar{s}}(e, \bar{s})\right|=\frac{\beta_{e} \varepsilon_{k}^{g} g}{\beta_{k} \varepsilon_{e}^{b} b}$. Hence the $\mathrm{s}=\bar{s}$ curve and the $\mathrm{t}=\bar{t}$ curve coincide as $\sigma$ tends toward zero. Invoking the definitions of $\beta_{e}, \beta_{k}, \varepsilon_{e}^{b}$ and $\varepsilon_{k}^{g}$ some rearrangement of terms yields $\left.\frac{\beta_{e} \varepsilon_{k}^{g} g}{\beta_{k} \varepsilon_{e}^{b} b}=\frac{p_{e} p_{z} z+\left(p_{e}+t\right) x}{\left(p_{e}+t\right)\left[\left(p_{z}+s\right) z+x\right]} \in\right] 0,1[$.

\section{Proof of Result 5 (excluding welfare changes)}

Starting from the system $\left(1^{\prime}\right)-\left(10^{\prime}\right)$ in the proof of Result 4 we derive the effect of the exogenous shocks in the parameters $\hat{p}_{e}, \hat{g}$ and $\hat{b}$ on the endogenous variables.

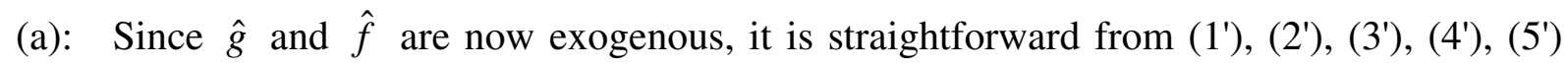
and $\left(10^{\prime}\right)$ that

$$
\hat{k}_{g}=\frac{1}{\varepsilon_{k}^{g}} \hat{g}, \quad \hat{k}_{x}=-\frac{\alpha_{g}}{\left(1-\alpha_{g}\right) \varepsilon_{k}^{g}} \hat{g}, \quad \hat{p}_{k}=\frac{\alpha_{g} \varepsilon_{k k}^{x}}{\left(1-\alpha_{g}\right) \varepsilon_{k}^{g}} \hat{g}, \quad \hat{x}_{s}=-\frac{\alpha_{g} \varepsilon_{k}^{x}}{\left(1-\alpha_{g}\right) \varepsilon_{k}^{g}} \hat{g}
$$

holds and, in addition,

$$
\hat{e}=\frac{1}{\varepsilon_{e}^{f}} \hat{b} \quad \text { and } \quad \hat{z}=\left(1-\alpha_{b}\right) \hat{g}+\alpha_{b} \hat{b}
$$


(b): Consider $\hat{x}_{s}$ from (A11) and $\hat{e}$ from (A12) in (6') to obtain, after some rearrangement of terms,

$$
\hat{x}=-\frac{1}{\alpha_{x} \varepsilon_{e}^{b}} \hat{b}-\frac{\alpha_{g}\left(1+\alpha_{x}\right) \varepsilon_{k}^{x}}{\alpha_{x}\left(1-\alpha_{g}\right) \varepsilon_{k}^{g}} \hat{g}-\frac{1}{\alpha_{x}} \hat{p}_{e} .
$$

(c): Consider $\hat{x}$ from (A13) and $\hat{z}$ from (A12) in (7') to obtain, after some rearrangement of terms,

$$
\hat{p}_{z}=-\frac{\beta_{e}}{\alpha_{x} \varepsilon_{e}^{b} \sigma} \hat{b}-\frac{\beta_{k}}{\alpha_{x} \varepsilon_{k}^{g} \sigma} \hat{g}-\frac{1}{\alpha_{x} \sigma} \hat{p}_{e} .
$$

(d): Consider $\hat{e}$ from (A12) and $\hat{p}_{z}$ from (A14) in (8') to obtain, after some rearrangement of terms,

$$
\hat{t}=-\frac{\beta_{e}+\alpha_{x} \varepsilon_{e e}^{b} \sigma}{\alpha_{x} \alpha_{t} \varepsilon_{e}^{b} \sigma} \hat{b}-\frac{\beta_{k}}{\alpha_{x} \alpha_{t} \varepsilon_{k}^{g} \sigma} \hat{g}-\frac{1+\alpha_{x}\left(1-\alpha_{t}\right) \sigma}{\alpha_{x} \alpha_{t} \sigma} \hat{p}_{e} .
$$

(e): Consider $\hat{k}_{g}$ and $\hat{p}_{k}$ from (A11) and $\hat{p}_{z}$ from (A14) in (9') to obtain, after some rearrangement of terms, $\hat{s}=\frac{\beta_{D}}{\alpha_{s} \alpha_{x} \varepsilon_{k}^{g} \sigma} \hat{g}+\frac{\left(1-\alpha_{s}\right) \beta_{e}}{\alpha_{s} \alpha_{x} \varepsilon_{e}^{b} \sigma} \hat{b}+\frac{\left(1-\alpha_{s}\right)}{\alpha_{s} \alpha_{x} \sigma} \hat{p}_{e}$.

\section{Welfare costs of variations in policy parameters and in the fossil fuel price}

The marginal change in the consumer's utility $u=U(x, z)$ is $d u=U_{x} x \hat{x}+U_{z} z \hat{z}$. The firstorder conditions of maximizing utility subject to the budget constraint (11) are $U_{x}=\lambda>0$ and $U_{z}=\lambda p_{z}$, where $\lambda$ denotes the marginal utility of income. Inserting these conditions in $d u=U_{x} x \hat{x}+U_{z} z \hat{z}$ yields $\frac{d u}{\lambda}=x \hat{x}+p_{z} z \hat{z}$ and making use of $\hat{x}=\hat{z}+\sigma \hat{p}_{z}$ from (7') gives us

$$
\frac{d u}{\lambda}=x \sigma \hat{p}_{z}+\left(p_{z} z+x\right) \hat{z}=x \sigma \hat{p}_{z}+y \hat{z} \quad \text { where } y:=p_{z} z+x .
$$

\section{(I) Parameter variations under conditions of Result 5}

(Ia) $\hat{g} \neq 0$ and $\hat{e}=\hat{p}_{e}=0$. Insert $\hat{p}_{z}=-\frac{\beta_{k}}{\alpha_{x} \varepsilon_{k}^{g} \sigma} \hat{g}$ from (A14) and $\hat{z}=\left(1-\alpha_{b}\right) \hat{g}$ from (A12) into (A16) to obtain $\frac{d u}{\lambda}=\left[\left(1-\alpha_{b}\right) y-\frac{\beta_{k} x}{\alpha_{x} \varepsilon_{k}^{g}}\right] \hat{g}$. Use the definitions of $\alpha_{b}, \alpha_{x}, \beta_{k}$ and $\varepsilon_{k}^{g}$ to turn the last equation into

$$
\frac{d u}{\lambda d g}=-s \quad \text { or, alternatively, } \quad\left(\frac{\hat{u}}{\hat{g}}\right)_{\bar{e}, p_{e}}=-\frac{g \lambda s}{u}<0 .
$$


(Ib) $\hat{e} \neq 0$ and $\hat{g}=\hat{p}_{e}=0$. Insert $\hat{p}_{z}=-\frac{\beta_{e}}{\alpha_{x} \sigma} \hat{e}$ and $\hat{z}=\alpha_{f} \varepsilon_{e}^{b} \hat{e}$ from (A12), (A14) and (3') into (A16) to obtain $\frac{d u}{\lambda}=\left[\alpha_{f} \varepsilon_{e}^{b} y-\frac{\beta_{e} x}{\alpha_{x}}\right] \hat{e}$. Use the definitions of $\alpha_{b}, \alpha_{x}, \beta_{e}$ and $\varepsilon_{e}^{b}$ to turn the last equation into

$$
\frac{d u}{\lambda d e}=t \quad \text { or, alternatively, } \quad\left(\frac{\hat{u}}{\hat{e}}\right)_{\bar{s}, p_{e}}=\frac{e \lambda t}{u}>0 .
$$

(Ic) $\hat{p}_{e} \neq 0$ and $\hat{e}=\hat{g}=0$. Insert $\hat{p}_{z}=-\frac{1}{\alpha_{x} \sigma} \hat{p}_{e}$ and $\hat{z}=0 \cdot \hat{e}$ from (A12), (A14) and (3') into (A16) to obtain

$$
\frac{d u}{\lambda d p_{e}}=-e \quad \text { or, alternatively, } \quad\left(\frac{\hat{u}}{\hat{p}_{e}}\right)_{\bar{e}, \bar{g}}=-\frac{\lambda p_{e} e}{u}<0 .
$$

\section{(II) Parameter variations under conditions of Result 4}

Consider first $\hat{s} \neq 0$ and $\hat{t}=\hat{p}_{e}=0$. As shown in Result 4 (and in Table 2), $\hat{s}$ leads to the changes $\left(\frac{\hat{g}}{\hat{s}}\right)_{\bar{t}, p_{e}}>0$ and $\left(\frac{\hat{e}}{\hat{s}}\right)_{\bar{t}, p_{e}}<0$, in $g$ and $e$ when $\hat{t}=\hat{p}_{e}=0$. We can therefore determine the impact of $\hat{s}$ on utility by the sum of the (partial) effects $\left(\frac{\hat{g}}{\hat{s}}\right)_{\bar{t}, p_{e}}$ and $\left(\frac{\hat{e}}{\hat{s}}\right)_{\bar{t}, p_{e}}$ on utility. In other words, (i) we know the effects of a policy change $\hat{s} \neq 0$ with $\hat{t}=\hat{p}_{e}=0$ on $e$ and $g$, (ii) we can consider these effects as having been generated by autonomous changes in the policy parameters $e$ and $g$ under the quantity-based mixed policy $(\bar{e}, \bar{g})$ and (iii) we finally assess the welfare effect of the changes in $e$ and $g$ via (A17) and (A18). Formally,

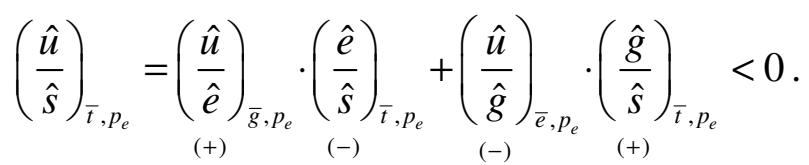

Following the same procedure as above $\hat{t} \neq 0$ and $\hat{s}=\hat{p}_{e}=0$ yields

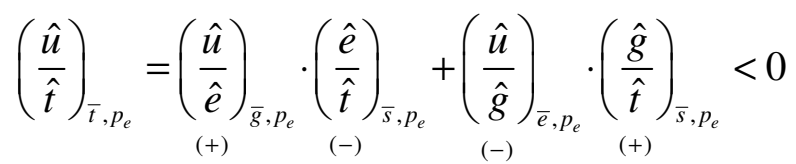

and $\hat{p}_{e} \neq 0$ and $\hat{s}=\hat{t}=0$ yields

$$
\left(\frac{\hat{u}}{\hat{p}_{e}}\right)_{\bar{s}, \bar{t}}=\underset{(+)}{\left(\frac{\hat{u}}{\hat{e}}\right)_{\bar{g}, p_{e}}} \cdot\left(\frac{\hat{e}}{\hat{p}_{e}}\right)_{\bar{s}, \bar{t}}+\underset{(-)}{\left(\frac{\hat{u}}{\hat{g}}\right)_{\bar{e}, p_{e}}} \cdot \underset{(+?)}{\left(\frac{\hat{g}}{\hat{p}_{e}}\right)_{\bar{s}, \bar{t}} .}
$$

Sufficient for $\left(\hat{u} / \hat{p}_{e}\right)_{\bar{s}, \bar{t}}<0$ is the condition given in Result 4 iid. 